\title{
Ion Source Development for Ultratrace Detection of Uranium and Thorium
}

\author{
Y. Liu ${ }^{1}$, J. C. Batchelder ${ }^{1,2}$, A. Galindo-Uribarri ${ }^{1,3}$, R. Chu ${ }^{1,3}$, S. Fan ${ }^{1,3}$ \\ E. Romero-Romero ${ }^{1,3}$, D. W. Stracener ${ }^{1}$ \\ ${ }^{1}$ Physics Division, Oak Ridge National Laboratory, Oak Ridge, TN 37831, USA \\ ${ }^{2}$ Oak Ridge Associated Universities, Oak Ridge, TN 37831, USA \\ ${ }^{3}$ Departmenty of Physics and Astronomy, University of Tennessee, Knoxville, TN 37966, USA
}

\begin{abstract}
Efficient ion sources are needed for detecting ultratrace $U$ and Th impurities in a copper matrix by mass spectrometry techniques such as accelerator mass spectrometry (AMS). Two positive ion sources, a hot-cavity surface ionization source and a resonant ionization laser ion source, are evaluated in terms of ionization efficiencies for generating ion beams of $U$ and $T h$. The performances of the ion sources are characterized using uranyl nitrate and thorium nitrate sample materials with sample sizes between $20-40 \mu \mathrm{g}$ of $\mathrm{U}$ or Th. For the surface ion source, the dominant ion beams observed are $\mathrm{UO}^{+}$or $\mathrm{ThO}^{+}$and ionization efficiencies of $2-4 \%$ have been obtained with W and Re cavities. With the laser ion source, three-step resonant photoionization of $U$ atoms has been studied and only atomic $U$ ions are observed. An ionization efficiency of about $9 \%$ has been demonstrated. The performances of both ion sources are expected to be further improved.
\end{abstract}




\section{Introduction}

Ultrasensitive analytical techniques are required for qualitative and quantitative analysis of the unwanted impurities in the materials constructing the detectors for ultra low-background experiments. A specific example is the Majorana Demonstrator (MJD) experiment [1] designed to search for neutrinoless double-beta-decay of the isotope ${ }^{76} \mathrm{Ge}$. The MJD is a $40-\mathrm{kg}$ detector array made from enriched ${ }^{76} \mathrm{Ge}$ and natural germanium and is being built to demonstrate the feasibility of a future one-ton-scale experiment. A specific goal of MJD is to achieve the ultralow backgrounds, about one count per ton-year in the region of interest, required for the larger experiment. In order to achieve these backgrounds, the MJD detector is constructed and operated deep underground to avoid background from cosmic rays and the detector mounts and cryostats are made from ultra-pure copper electroformed and machined underground. Monte Carlo simulations indicate that uranium (U) and thorium (Th) impurities in this copper are expected to be the dominant sources of background and must be below the levels of a few $10^{-14}$ by weight for ${ }^{238} \mathrm{U}$ and ${ }^{232} \mathrm{Th}$.

We have proposed to use accelerator mass spectrometry (AMS) to evaluate the U and Th impurities in the ultra-pure copper materials for MJD. AMS is presently the most sensitive and well established technique for trace elemental and isotope analysis, in comparison with other mass spectrometry techniques such as inductively coupled plasma mass spectrometry (ICP-MS), resonant ionization mass spectrometry (RIMS), and thermal ionization mass spectrometry (TIMS). It has been previously used to detect rare actinide isotopes with detection limits of $10^{-11}$ $10^{-12}$ isotope abundance ratios [2]. A major factor that limits the AMS sensitivity is the low ionsource efficiency, which is important for trace analysis. Cs-sputter negative ion sources are 
typically used for AMS and their ionization efficiencies are reported to be $0.01-0.1 \%$ for $\mathrm{U}$ [3$6]$.

This work is motivated by the need for more efficient ion sources for AMS analysis of U and Th impurities in a copper matrix at the trace levels required for the MJD experiment. Our approach is to use highly efficient positive ion sources which promise much higher ionization efficiencies for $\mathrm{U}$ and $\mathrm{Th}$ than Cs-sputter negative sources. This approach takes advantage of the available expertise and infrastructure of the former Holifield Radioactive Ion Beam Facility (HRIBF) [7] at the Oak Ridge National Laboratory (ORNL) where various positive ion sources were developed for the generation of ion beams of exotic nuclei. The positive ions were converted to negative ions by charge exchange and then sent to the 25-MV Tandem accelerator for acceleration to the desired energies for subsequent nuclear studies. The infrastructure at HRIBF is unique for AMS applications. The 25-MV tandem accelerator has the highest terminal voltage in the world. Charge stripping can be accomplished with thin carbon foils or a dilute gas in the terminal followed by a $180^{\circ}$ magnet for charge-state selection. A number of ion sources are available to provide negative ion beams, including Cs-sputter negative ion sources and positive ionization sources in combination with a charge exchange cell. The capability of the HRIBF infrastructure for AMS application has been proven in a recent work [8] where fully stripped ${ }^{36} \mathrm{Cl}$ ions were obtained with the $25-\mathrm{MV}$ tandem, thus effectively removing the ${ }^{36} \mathrm{~S}$ isobars and pushing the detection limit of ${ }^{36} \mathrm{Cl} / \mathrm{Cl}$ to a few times $10^{-16}$. More information about the AMS system at HRIBF can be found in Ref. [9].

Two positive-ion sources are considered: a hot-cavity surface ionization source (HCSIS) and a hot-cavity resonant ionization laser ion source (RILIS). High temperature cavity ion sources have been used for more than 30 years at isotope separator on-line (ISOL) facilities for 
producing radioactive ion beams of elements of relatively low ionization potentials [10-12]. More recently, cavity-type surface ionization sources [13-18] are employed for TIMS systems for the purpose of improving the ionization efficiency and thus detection limits of low-level isotope analyses of elements such as $\mathrm{U}, \mathrm{Th}$, and $\mathrm{Pu}$ for which the conventional filament-type thermal ionization sources have very poor efficiencies. Measured ionization efficiencies of 3$8.5 \%$ for $\mathrm{U}[13,14,16,18]$ and $1-3 \%$ for Th $[13,15]$ have been reported for those sources. The RILIS is developed for the production of isobarically pure radioactive ion beams (RIBs) at the ISOL facilities. It has been used, or demonstrated the ability, to provide ion beams of more than 40 elements in the periodic table with ionization efficiencies up to $40 \%$ [19,20]. An important advantage of the RILIS is its high selectivity for suppressing unwanted interfering ions. The two positive sources have been evaluated for their efficiencies in generating ion beams of $\mathrm{U}$ and $\mathrm{Th}$. The design features and operating principles of the sources, the experimental setup, and the experimental results will be presented.

\section{Hot-cavity surface ionization source}

Atoms or molecules impinging on a hot metal surface may be emitted as atoms or ions, depending on the work function, $\Phi$, of the metal surface and the first ionization potential, IP, of the atom or molecule. The degree of ionization, $\alpha$, is given by the Saha-Langmuir equation

$$
\alpha=\frac{n_{i}}{n_{0}}=\frac{g_{i}}{g_{0}} \exp \left(\frac{\Phi-I P}{k T}\right),
$$

where $n_{i}$ and $n_{0}$ are the concentrations of the ions and neutrals leaving the surface and $g_{i}$ and $g_{0}$ are the statistical weights of the ionic and atomic states, respectively, $k$ is the Boltzmann's 
constant, and $T$ is the temperature of the surface. The surface ionization efficiency, $\beta$, is defined as

$$
\beta=\frac{n_{i}}{n_{i}+n_{0}}=\frac{\alpha}{1+\alpha} .
$$

From Eqs. (1) and (2) it is evident that high ionization efficiencies can be obtained for high work-function materials and low ionization-potential species such as the alkali metals. For the elements such as $\mathrm{U}(\mathrm{IP}=6.194 \mathrm{eV})$ and $\mathrm{Th}(\mathrm{IP}=6.307 \mathrm{eV})$ for which $\mathrm{IP}>\Phi$, the surface ionization process is much less efficient. For example, the efficiency of ionizing $\mathrm{U}$ and Th on a tungsten surface $(\Phi=4.54 \mathrm{eV})$ at $\mathrm{T}=3000 \mathrm{~K}$ is only about $0.13 \%$ and $0.09 \%$, respectively.

The degree of ionization predicted by Eq. (1) assumes that the ions are extracted as soon as they are formed. It has been observed that the efficiency of a surface ionization source can be substantially increased by the use of a high-temperature cavity with a small extraction hole [21,22]. For instance, ionization efficiencies of 4 - 39\% were measured [23] for U with tantalum, tungsten, and rhenium cavities at about $2900 \mathrm{~K}$. The mechanisms for the enhancement have been extensively studied $[10,12,15,21-26]$ and will not be presented here in detail. Briefly, the neutral species undergo significantly higher number of wall collisions with the hot-cavity configuration and thus have a much larger probability to be ionized. In addition, a thermal plasma, consisting of surface ionized positive ions and thermionic electrons emitted from the hot walls, could form inside the hot-cavity. The plasma may reach near thermodynamic equilibrium with the cavity wall. Due to the high thermionic electron density at the wall, a negative plasma sheath potential with respect to the wall is created so that the positive ions can be confined to the plasma, preventing ion-wall collisions and thus ion losses. Therefore, the 'thermal' ionization efficiency can be much higher than that of surface ionization. 


\subsection{Description of the source}

A cross sectional side view of the HCSIS assembly is displayed in Fig. 1, which shows the principal components: the hot-cavity ionizer, the sample tube, the graphite housing, and the extraction-electrode of the ion source. The hot-cavity ionizer is a cylindrical cavity of 3-mm inner diameter (ID), 30-mm long and 1-mm wall thickness. High work function and refractory materials such as $\mathrm{Ta}, \mathrm{W}$, and Re are tested as the ionizer material. The ionizer is connected to a closed-end Ta tube of 8.5-mm inner diameter and about $100 \mathrm{~mm}$ long, in which samples are placed at selected locations depending on the sample materials. The ionizer and the sample tube are heated resistively by passing a current through the tubular structure and can be heated to temperatures exceeding $3000 \mathrm{~K}$ in the ionizer. The sample materials in the sample tube are heated along with the sample tube and the ionizer. The volatile species effuse from the sample tube into the ionizer where they are ionized by surface ionization. The ions are extracted by the conical electrode.

\subsection{Experimental results}

The ion source was evaluated at the on-line test facility (OLTF) [7]. Positive ions extracted from the ion source were accelerated to about $40 \mathrm{keV}$ energies and focused into a $90^{\circ}$ dipole magnet with a resolving power of $\sim 2000$ for mass separation. The mass-selected ion beam current was measured with a Faraday cup after the magnet. The total ion current extracted from the ion source was also monitored with a Faraday cup before the dipole magnet.

The sample materials were made from $1000 \mathrm{ppm} \mathrm{U}$ or Th atomic absorption (AA) standard solutions, which contain $U$ and Th in a nitric acid matrix in the form of uranyl nitrate and thorium nitrate, respectively. Small samples containing about $40 \mu \mathrm{g}$ of $\mathrm{U}$ or Th atoms were 
used to evaluate the source. To make a sample, the selected $40 \mu \mathrm{L}$ AA solution was dried on a thin Ti foil (0.0005 inch in thickness and about $5 \times 6 \mathrm{~mm}$ in size) and then wrapped in the foil. The sample was placed in the sample tube of the source and heated when the ionizer and sample tube are heated. The sample tube was on average several hundred degrees colder than the ionizer. Ideally, the temperatures of the sample and the ionizer should be independently controlled. However, this could not be achieved with the present source assembly which was designed for on-line production of radioactive ion beams.

Figure 2 shows the mass spectra of the ion beams obtained with a $\mathrm{W}$ ionizer at two source heating currents: (a) $420 \mathrm{~A}$ and (b) $500 \mathrm{~A}$, which correspond to ionizer temperatures of approximately $2200 \mathrm{~K}$ and $3000 \mathrm{~K}$, respectively. The measurement was conducted with one 40 $\mu \mathrm{g} \mathrm{U}$ sample and one $40 \mu \mathrm{g}$ Th sample in the transfer line. As noted, the operating temperatures for the Th samples were much higher than those for the U samples. At a source heating current of $420 \mathrm{~A}, \mathrm{ThO}^{+}$and $\mathrm{Th}^{+}$ions were well below $1 \mathrm{nA}$, while at $500 \mathrm{~A}$ they were the dominant ions. This is consistent with the fact that thorium and thorium oxides have higher melting points and lower vapor pressures than uranium and uranium oxides [27].

As the ionizer was heated to operational temperatures, the ion beams extracted from the source were monitored. As shown in Fig. 2, molecular ions of $\mathrm{UO}^{+}$and $\mathrm{ThO}^{+}$were always observed first and were the dominant ions. The relative intensities of the atomic $\mathrm{U}^{+}$and $\mathrm{Th}^{+}$ions increased at higher temperatures. This behavior, together with the ionization potentials of the atomic and molecular species [U (IP: $6.19 \mathrm{eV}$ ), UO (IP: $6.03 \mathrm{eV}), \mathrm{UO}_{2}$ (IP: $6.13 \mathrm{eV}$ ), Th (IP: $6.31 \mathrm{eV}$ ), and ThO (IP: $6.6 \mathrm{eV}$ )], indicates that the volatile oxides, instead of metal $\mathrm{U}$ and $\mathrm{Th}$, were released from the nitrate samples. At higher temperatures, the oxides may begin to dissociate into the atomic components. $\mathrm{ThO}_{2}{ }^{+}$ions were not observed due to its high IP (8.7 eV). 


\subsection{Ionization efficiencies}

The ionization efficiency of the HCSIS was measured with quantified nitrate samples containing $\sim 10^{17} \mathrm{U}$ or Th atoms per sample. The sample was placed in the sample tube and heated as the ionizer and sample tube were gradually heated to operating temperatures. The mass-selected ion $\left(\mathrm{UO}^{+}\right.$or $\left.\mathrm{ThO}^{+}\right)$current extracted from the source was continuously recorded until it dropped below 2-3 nA while the ionizer and sample tube were at high temperatures and further heating did not increase the ion current. This was considered as the indication that the sample materials were mostly evaporated and effused out of the source. The ionization efficiency was then obtained as the ratio of the integrated total number of detected ions to the total number of neutral atoms in the sample. Table 1 gives the measured efficiencies for three ionizer materials: $\mathrm{Ta}(\Phi=4.25 \mathrm{eV}), \mathrm{W}(\Phi=4.54 \mathrm{eV})$, and $\operatorname{Re}(\Phi=5.1 \mathrm{eV})$. Work is in progress to complete the efficiency measurements for $U$ and Th with the three materials. As expected, cavities of higher work-function materials give higher ionization efficiencies. The best cavity material should be Re. However, rhenium is expensive and difficult to machine. The Re cavity tested was formed with a thin Re foil and was good for one-time use. Sources with a solid Re cavity made from a Re rod will be available soon.

The above results are comparable to the efficiencies for $\mathrm{U}$ and $\mathrm{Th}$ with hot-cavity sources in recent literature [13-18], but well below the reported [23] U efficiencies of 4.5(7)\%, 15(5)\%, and 39(6)\% with Ta, W, and Re cavities, respectively. This suggests that there could be a large room for improvement. We will explore a number of means to enhance the HCSIS efficiency. For example, the ions were distributed between 2-3 species of oxides and atom. Duan et al. $[13,14]$ have observed that adding graphite into the cavity could convert most of the uranium oxide ions to uranium metal ions and increase the ionization efficiency of the sample by a factor 
of $2[13,14]$. Kirchner reported that increasing the neutral density in the cavity, by feeding in a non-ionizing gas such as Xe, could increase the ionization efficiency by a factor of $\sim 5$ [23]. This effect is ascribed to the reduction of the mean free path and thus the probability of wall recombination of the ions. These enhancement mechanisms will be investigated in the near future.

\section{Resonant ionization laser ion source}

A resonant ionization laser ion source (RILIS) uses multiple lasers to stepwise, resonantly excite atomic species to higher energy levels followed by ionization in the last transition. Since each element has its own unique set of atomic energy levels, the resonant excitation and ionization process can provide nearly $100 \%$ elemental selectivity and can be highly efficient. Such high Z-selectivity is necessary for the production of isobarically pure RIBs. It is also a key advantage of the RILIS for ultra-trace analysis applications. The RILIS used for the present study was developed at ORNL for the former HRIBF research program $[20,28]$. It has two main components: a hot-cavity ion source and a laser system. The hot-cavity ion source is the same hot-cavity surface ionization source described above (Fig. 1), except here surface ionization is undesired as it may lower the selectivity of the RILIS. Hence, a tantalum ionizer ( $3 \mathrm{~mm}$ ID and $30 \mathrm{~mm}$ long) is typically used. The laser system consists of three pulsed, tunable Ti:Sapphire lasers, each pumped by a Q-switched Nd:YAG laser at $532 \mathrm{~nm}$ with average output power of $19 \mathrm{~W}$ at $10 \mathrm{kHz}$ pulse repetition rate. The Ti:Sapphire lasers use a diffraction grating for wavelength selection and are continuously tunable between $720 \mathrm{~nm}$ and $960 \mathrm{~nm}$. The fundamental laser outputs can be frequency doubled, tripled, and quadrupled to obtain laser wavelengths in the blue to UV region. The laser system can provide up to three-step and three- 
photon ionization of elements with IP $\leq \sim 9 \mathrm{eV}$. More detailed information on the RILIS can be found in $[20,28]$.

The RILIS was installed at the Injector for Radioactive Ion Species 2 (IRIS2) [28]. The experimental setup was similar to that for HCSIS. Laser beams were collimated and focused into the hot-cavity ionizer of the source from the extraction electrode side, ionizing the atoms of interest before they escape out of the ionizer. The ions were extracted out by the extraction electrode and accelerated to $15-18 \mathrm{keV}$ energies and mass separated by a dipole magnet system with a nominal resolving power of $1000: 1$. The mass-selected ion beam current was measured with a Faraday cup.

\subsection{Resonant ionization of $\mathrm{U}$}

Laser ionization of $U$ has been studied for applications such as isotope separation and isotope ratio measurement by RIMS. The earlier works mostly employed tunable dye lasers. With the emerging of all-solid-state tunable lasers such as Ti:Sapphire and diode lasers, spectroscopy studies to develop efficient resonant ionization schemes for actinide elements, including $U$ and $T h$, using these solid-state lasers have recently been carried out by the LARISSA group at the University of Mainz [29-33]. Their studies revealed numerous atomic energy levels in $U$ that were previously not known and identified excitation and ionization schemes optimized for Ti:Sapphire or diode lasers. Ionization efficiencies on the order of $0.04 \%$ for $\mathrm{U}$ isotopes were also obtained [33]. It is therefore of interest to explore possible improvements in the efficiency. 
The work presented here is our first study on ionization of U in a RILIS. We selected several three-step, three-photon ionization schemes using at first the results of the LARISSA group. As illustrated in Fig. 3, they all utilized the first step of exciting $U$ atoms from the first excited level $5 \mathrm{f}^{3} 6 \mathrm{~d} 7 \mathrm{~s}^{2}{ }^{5} \mathrm{~K}_{5}^{\circ}$ at $620.323 \mathrm{~cm}^{-1}$ to the excited state at $25348.972 \mathrm{~cm}^{-1}$ by the first photon $\left(\lambda_{1}=404.389 \mathrm{~nm}\right)$. From this level, two resonant transitions were selected: (1) to a level at $37412.181 \mathrm{~cm}^{-1}\left(\lambda_{2}=828.967 \mathrm{~nm}\right)$ or $(2)$ to a level at $38019.153 \mathrm{~cm}^{-1}\left(\lambda_{2}=789.255 \mathrm{~nm}\right)$. In the third step, the excited $U$ atoms were ionized by transitions to an autoionizing level above the ionization potential. The wavenumber of the third photon was scanned over a limited range of $300-500 \mathrm{~cm}^{-1}$ to search for relatively stronger autoionization transitions. The scheme on the right in Fig. 3 was found to be the best, with which about $50 \%$ more U ion current was obtained than the next best scheme tested. In this scheme, $\mathrm{U}$ atoms are excited from the excited state at $620.323 \mathrm{~cm}^{-1}$ above the ground state, to the resonant level at $25348.972 \mathrm{~cm}^{-1}$ by the first photon $\left(\lambda_{1}=404.389 \mathrm{~nm}\right)$, then promoted to the level at $37412.181 \mathrm{~cm}^{-1}$ by the second photon $\left(\lambda_{2}=\right.$ $828.967 \mathrm{~nm})$, and subsequently ionized by the third photon $\left(\lambda_{3}=782.33 \mathrm{~nm}\right)$ via excitation to an autoionizing level at $50194.49 \mathrm{~cm}^{-1}$. The first photons were frequency-doubled from the fundamental laser output. The laser power for each step before injection into the ion source was measured to be about $300 \mathrm{~mW}$ for the first step after frequency doubling, and about $1.2 \mathrm{~W}$ for both $2^{\text {nd }}$ and $3^{\text {rd }}$ steps. All three transitions were found to be well saturated with the available laser power. This scheme was used for the efficiency measurement presented below.

\subsection{Ionization efficiency}

The ionization efficiency of the RILIS for $U$ was measured with a smaller sample containing $\sim 5 \times 10^{16} \mathrm{U}$ atoms wrapped in a thin $\mathrm{Zr}$ foil $(0.001$ " thick, $\sim 5 \times 6 \mathrm{~mm})$. The 
measurement procedure was almost the same as that described above for the HCSIS, except that here $\mathrm{U}$ atoms released from the sample were ionized by the laser beams and the mass-selected $\mathrm{U}^{+}$ ion current was recorded. An overall ionization efficiency of about $9 \%$ was obtained. This value is more than double the efficiencies obtained so far with the HCSIS. Throughout the measurement, surface ionized $\mathrm{U}^{+}$ions were checked frequently by blocking all the laser beams and less than $0.4 \mathrm{nA}$ of the surface ions were observed even at very high ionizer temperatures $(>2500 \mathrm{~K})$.

This is our first result with the RILIS. Higher efficiencies can be expected by several means. As shown in Fig. 3, the first excitation step involved the $\mathrm{U}$ atoms in the ${ }^{5} \mathrm{~K}^{\circ}{ }_{5}$ first excited state. This excited state is less thermally populated than the ground state at the operating temperatures. For example, up to $3500 \mathrm{~K}$, less than $40 \%$ of the $\mathrm{U}$ atoms will be in the ${ }^{5} \mathrm{~K}^{\circ}{ }_{5}$ state, while the remaining $\mathrm{U}$ atoms will occupy the ground state. Hence, ionization schemes involving excitations of $U$ atoms from the ground state in the first step could be more efficient. Such schemes have been reported [29] and will be evaluated with our RILIS. In addition, many atomic spectral lines have been observed in $U$ [31-33]. Only a small fraction of the possible combinations of the excitation and ionization transitions were examined in this work. Effort is under way to improve the experimental setup so that the wavenumbers of the second and third lasers can be scanned over a much wider range $\left(\geq 3000 \mathrm{~cm}^{-1}\right)$ to search for more efficient excitation and ionization ladders. Finally, non-resonant ionization in the last step by the highpower pump laser at $532 \mathrm{~nm}$ is shown in more and more cases to yield higher efficiencies than resonant ionization [19]. This feasibility will also be explored for $\mathrm{U}$. 


\section{Discussion}

For AMS analysis, positively charged $\mathrm{U}$ and Th atomic or molecular ions must be converted to negative ions for injection into the tandem accelerator. The conversion efficiency may play a critical role in the use of positive ion sources for tandem-accelerator-based AMS. To obtain negative ions, the positive ion beam can be injected into a charge-exchange cell (CEC) containing an alkali or alkaline vapor of appropriate density where the initially positive ions are converted into negative ions through charge-exchange collisions with the vapor atoms. To our knowledge, there have been no published data on the charge-exchange efficiencies of $U$ and $T h$ positive ions. It is well known that the charge exchange is a two-step process and the yield of negative ions depends primarily on the collision energy, the electron affinity (EA) of the projectile element, and the IP and density of the exchange vapor [34]. It is generally true that higher charge-exchange efficiencies can be expected for higher projectile EA values. $\mathrm{U}$ and $\mathrm{Th}$ have relatively low electron affinities, as predicted to be $0.3-0.37 \mathrm{eV}$ for $\mathrm{U}^{-}$and $0.37 \mathrm{eV}$ or 0.5 $\mathrm{eV}$ for $\mathrm{Th}^{-}[35,36]$. Such low EA value is clearly a disadvantage for charge conversion. However, positive ion sources in combination of a CEC have been extensively used at ORNL to generate many negative radioactive ion beams for post-acceleration with the $25-\mathrm{MV}$ tandem accelerator. We have observed that the overall efficiency of the charge-exchange process is not only determined by the EA value but also strongly depends on the nature of the projectile-vapor combination and the projectile energy. For example, using a Cs CEC, the highest efficiencies for producing $\mathrm{As}^{-}(\mathrm{EA}=0.81 \mathrm{eV}), \mathrm{Sn}^{-}(\mathrm{EA}=1.22 \mathrm{eV}), \mathrm{Se}^{-}(\mathrm{EA}=2.02 \mathrm{eV}), \mathrm{Br}^{-}(\mathrm{EA}=3.37 \mathrm{eV})$ and $\operatorname{Sr}^{-}(\mathrm{EA}=0.05 \mathrm{eV})$ beams are respectively $42 \%, 41 \%, 22 \%, 1.2 \%$, and $0.9 \%$, with optimum projectile energies ranging from $20 \mathrm{keV}$ to $50 \mathrm{keV}$. A charge exchange efficiency of $\sim 40 \%$ for $\mathrm{Ga}^{-}(\mathrm{EA}=0.30 \mathrm{eV})$ has been reported [37]. Heinemeier and Tykesson [38] obtained charge- 
exchange efficiencies of 2-6\% for low-EA elements such as $\mathrm{Be}^{-}\left(\mathrm{EA}=0.24 \mathrm{eV}\right.$, metastable), $\mathrm{B}^{-}$ $(\mathrm{EA}=0.28 \mathrm{eV})$ and $\mathrm{Fe}^{-}(\mathrm{EA}=0.25 \mathrm{eV})$, using $\mathrm{Na}$ or $\mathrm{Mg}$ vapors and at a fixed projectile energy of $20 \mathrm{keV}$. Based on these experimental results, we believe that efficiencies on the order of 5 $10 \%$ could be expected for converting positively-charged $\mathrm{U}$ and Th atomic ions to negative ions using a proper charge-exchanging vapor. We will investigate the optimal charge-exchanging conditions and measure the conversion efficiencies of $\mathrm{U}$ and $\mathrm{Th}$ positive ions in the near future.

Molecular $\mathrm{UO}^{+}$and $\mathrm{ThO}^{+}$ions can also be converted to negative ions by charge exchange, but from our experience they will likely dissociate into atomic species during the charge-exchange collisions and the overall conversion efficiency for negative ion formation will be lower than that of the atomic positive ions. Moreover, the resulting atomic negative-ion beam could have a large energy spread of up to several hundreds of $\mathrm{eV}$ due to the three-body kinematics of molecular breakup. Therefore, molecular $\mathrm{UO}^{+}$and $\mathrm{ThO}^{+}$beams are not preferred. We will focus on converting the molecules to atoms by means such as introducing graphite $[13,14]$ into the HCSIS ion source in the future.

To compare with Cs-sputter negative ion sources, it is noted that even with the preliminary RILIS ionization efficiency of $9 \%$ for $\mathrm{U}$ and a $1 \%$ charge-exchange efficiency, the overall efficiency of producing $\mathrm{U}^{-}$ion with the RILIS + CEC combination is comparable with that of Cs-sputter sources (0.01-0.1\% [3-6]), while the purity of the $\mathrm{U}^{-}$beam from the RILIS and CEC could be orders of magnitude better. Therefore, the AMS detection limit could still be substantially improved with the RILIS. 


\section{Summary}

In summary, two positive ion sources, hot-cavity surface ionization source (HCSIS) and resonant ionization laser ion source (RILIS), have been evaluated in terms of ionization efficiency for generating ion beams of $U$ and Th. For the HCSIS, the dominant ion beams observed were $\mathrm{UO}^{+}$or $\mathrm{ThO}^{+}$and ionization efficiencies of $2-4 \%$ have been obtained with $\mathrm{W}$ and Re cavities. Resonant ionization of $U$ in a RILIS has been obtained using three-step, threephoton ionization schemes. The laser-ionized ions were atomic $\mathrm{U}^{+}$ions and an ionization efficiency of $9 \%$ for $\mathrm{U}$ has been achieved. For U, the RILIS is shown to be more efficient than the HCSIS. Moreover, the RILIS is highly selective for suppressing interfering and background ions. Laser ionization of Th has not been conducted but will be done in the near future.

Work is still in progress to improve the efficiencies of both positive ion sources. Higher efficiencies with the HCSIS can be expected by optimizing the cavity material and geometry as well as the operating conditions. In addition, means to convert the molecular ions into atomic $\mathrm{U}$ and Th ions such has graphitization will be investigated. For the RILIS, we will continue the search for more efficient resonant schemes for $U$ and study alternative schemes such as the feasibility of non-resonant ionization in the last step with the high-power pump laser at $532 \mathrm{~nm}$.

The objective of this study is to detect ultra-trace amount of $U$ and Th impurities in a copper matrix. Our next step will focus on the performance of the HCSIS and RILIS with smaller $\mathrm{U}$ and Th nitrate materials down to nanogram and picogram sizes as well as copper samples mixed with small amount of $U$ and Th. The experimental setup is being modified with the addition of a channeltron detector for measuring low intensity ion currents. 


\section{Acknowledgements}

This material is based upon work supported by the U.S. Department of Energy, Office of Science, Office of Nuclear Physics and by the Laboratory Directed Research and Development

Program at Oak Ridge National Laboratory, managed by UT-Battelle, LLC, for the U.S. Department of Energy.

\section{References}

1. N. Abgrall, et al., Advances in High Energy Physics, Vol. 2014, Article ID 365432. http://dx.doi.org/10.1155/2014/365432.

2. P. Steier, et al., Nucl. Instr. and Meth. B 268 (2010) 1045-1049.

3. M. Srncik, et al., J. Envirn. Radioactivity 102 (2011) 614-619.

4. P. Steier, et al., Nucl. Instr. and Meth. B 266 (2008) 2246-2250.

5. M.A.C. Hotchkis, et al., Nucl. Instr. and Meth. B 172 (2000) 659-665.

6. X. Wang, et al., Nucl. Instr. and Meth. B 268 (2010) 2295-2299.

7. J. R. Beene, D. W. Bardayan, A. Galindo-Uribarri, C. J. Gross, K. L. Jones, J. F. Liang, W. Nazarewicz, D. W. Stracener, B. A. Tatum, R. L. Varner, J. Phys. G 38 (2011) 024002.

8. A. Galindo-Uribarri et al., Nucl. Instr. Meth. B 259 (2007) 123.

9. M. S. Janzen, A. Galindo-Uribarri, Y. Liu, G. Mills, E. Romero-Romero, D. W. Stracener, This Proceedings.

10. R. Kirchner, Nucl. Instr. and Meth.186 (1981) 275.

11. H. L. Ravn, Nucl. Instr. and Meth. B 70 (1992) 107.

12. R. Kirchner, Rev. Sci. Instrum. 67 (1996) 928. 
13. Y. Duan, E. P. Chamberlin, J. A. Olivares, Int. J. Mass Spectrom. Ion Processes 161 (1997) 27.

14. Y. Duan, R. E. Danen, X. Yan, R. Steiner, J. Cuadrado, D. Wayne, V. Majidi, and J. A. Olivares, J. Am. Soc. Mass Spectrom. 10 (1999) 1008.

15. D. M. Wayne, W. Hang, D. K. McDaniel, R. E. Fields, E. Rios, V. Majidi, Int. J. Mass Spectrometry 216 (2002) 41.

16. L.R. Riciputi, et al., Proc. IAEA-CN-98/25P, 2003, p.347.

17. S. Burger, L.R. Riciputi, S. Turgeon, D. Bostick, E. McBay, M. Lavelle, J. Alloys and Compounds 660 (2007) 444-445.

18. Zhai Li-hua, Deng Hu, Wei Guan-yi, Li Zhi-ming, Wang Chang-hai, Li Xue-song, Zhou Guo-qing, Su Yong-yang, Zhang Zi-binet al., Int. J. Mass Spectrometry 305 (2011) 45.

19. V. N. Fedosseev, Yu Kudryavtsev, V. I. Mishin, Phys. Scr. 85 (2012) 058104.

20. Y. Liu, Hyperfine Interact. 227 (2014) 85.

21. G. J. Beyer, E. Herrmann, A. Plotrowskl, V. I. Rmko, H Tyrrott, Nucl Instr and Meth. 96 (1971) 437.

22. P. G. Johnson, A. Bolson and C. M. Henderson, Nucl. Instr. and Meth. 106 (1973) 83.

23. R. Kirchner, Nucl. Instr. and Meth. A 292 (1990) 203.

24. M. Huyse, Nucl. Instr. and Meth. 215 (1983) 1.

25. M. Turek, A. Droz'dziel, K. Pyszniak, D. Maczka, and B. Słowin'ski, Rev. Sci. Instrum. 83 (2012) 023303.

26. M. Turek, Vacuum 104 (2014) 1.

27. Thermophysical Properties of Materials for Nuclear Engineering: A Tutorial and Collection of Data, IAEA-THPH, Vienna, 2008. 
28. Y. Liu, C.U. Jost, A.J. Mendez II, D.W. Stracener, C.L. Williams, C.J. Gross, R.K. Grzywacz, M. Madurga, K. Miernik, D. Miller, S. Padgett, S.V. Paulauskas, K.P. Rykaczewski, M. Wolinska-Cichocka, Nucl. Instr. and Meth. B 298 (2013) 5.

29. P.G. Schumann, K.D.A. Wendt, B.A. Bushaw, Spectrochimica Acta Part B 60 (2005) 402.

30. B. A. Bushaw, S. Raeder, S. L. Ziegler, K. Wendt, Spectrochimica Acta Part B 62 (2007) 485.

31. S. Raeder, S. Fies, T. Gottwald, C. Mattolat, S. Rothe, K. Wendt, Hyperfine Interact. 196 (2010) 71.

32. A. Hakimi, T. Fischbach, S. Raeder, N. Trautmann, K. Wendt, Hyperfine Interact. 216 (2013) 59 .

33. S. Raeder, Spurenanalyse von Aktiniden in der Umwelt mittels ResonanzionisationsMassenspektrometrie, Ph.D. thesis, University of Mainz, 2010.

34. G. D. Alton, Negative-Ion Formation and Processes and Sources, in Electrostatic Accelerators: Fundamentals and Applications, edited by R. Hellborg (Springer Verlag, Heidelberg, 2005), pp. 223-273.

35. S. G. Bratsch and J. J. Lagowski, Chem. Phys. Letters 107 (1984) 136.

36. W. E. Bunting and J. C. Ellenbogen, Phy. Rev. A 85 (2012) 062503.

37. R. L. Auble, AIP Conference Proceedings 475 (1999) 292.

38. J. Heinemeier and P. Tykesson, Revue de Physique Appliquée 12 (1977) 1471. 
Table 1. Measured ionization efficiencies of the HCSIS with different cavity materials.

\begin{tabular}{cccc}
\hline \multirow{2}{*}{ element } & \multicolumn{3}{c}{ Cavity Material } \\
\cline { 2 - 4 } & $\mathrm{Ta}$ & $\mathrm{W}$ & $\mathrm{Re}$ \\
\hline $\mathrm{U}$ & $0.9(1)$ & $3.0(8)$ & \\
$\mathrm{Th}$ & & $2.5(2)$ & $4.0(2)$ \\
\hline \hline
\end{tabular}

\section{Figure Captions:}

Fig. 1. Cross sectional side view of the hot-cavity surface ionization source (HCSIS) assembly.

Fig. 2. Mass spectra of $U$ and Th ions measured at source heating currents of (a) 420A and (b) 500A, at which the ionizer temperatures were approximately $2200 \mathrm{~K}$ and $3000 \mathrm{~K}$, respectively.

Fig. 3. Three-step, three-photon resonant ionization schemes for $U$ evaluated in the present work. 


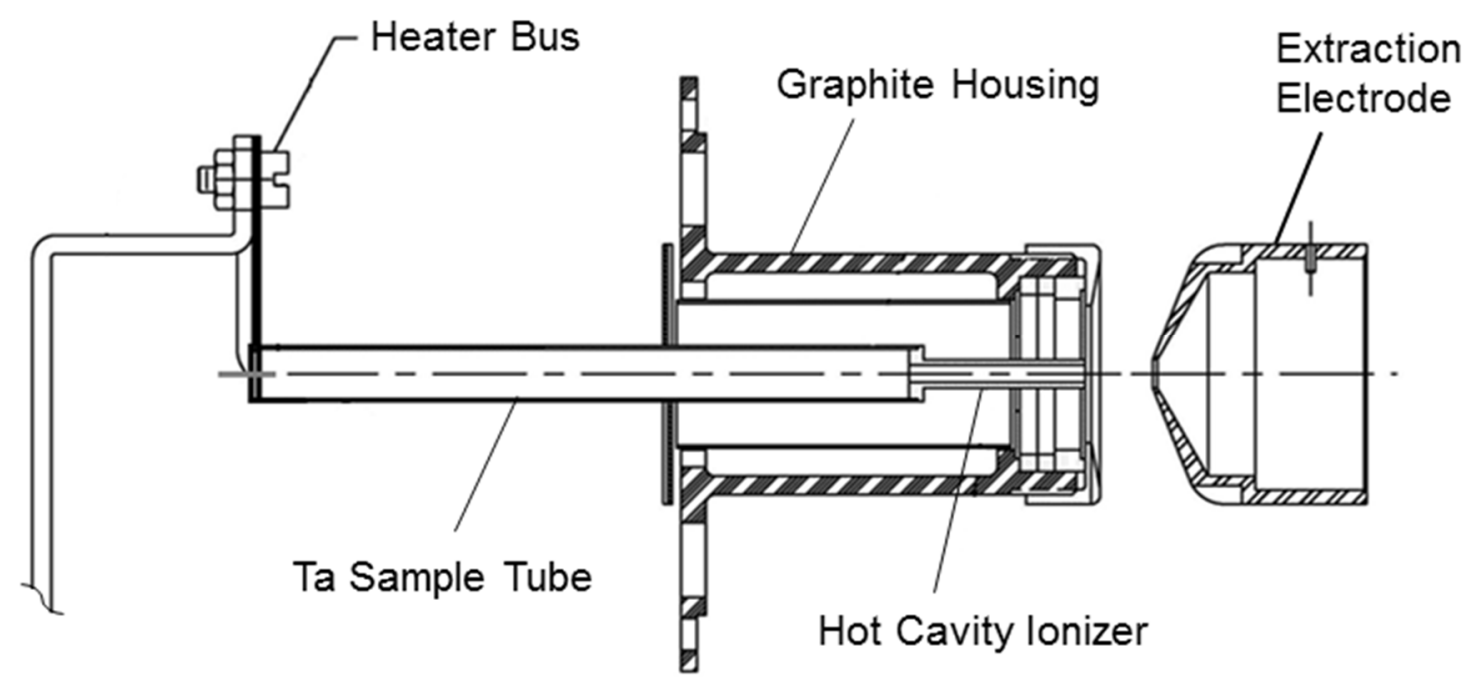

Figure 1

29

30

31

32

33

34

35

36

37

38

39

40

41

42

43

44

45

46

47

48

49

50

51

52

53

54

55

56

57

58

59

60

61

62

63 


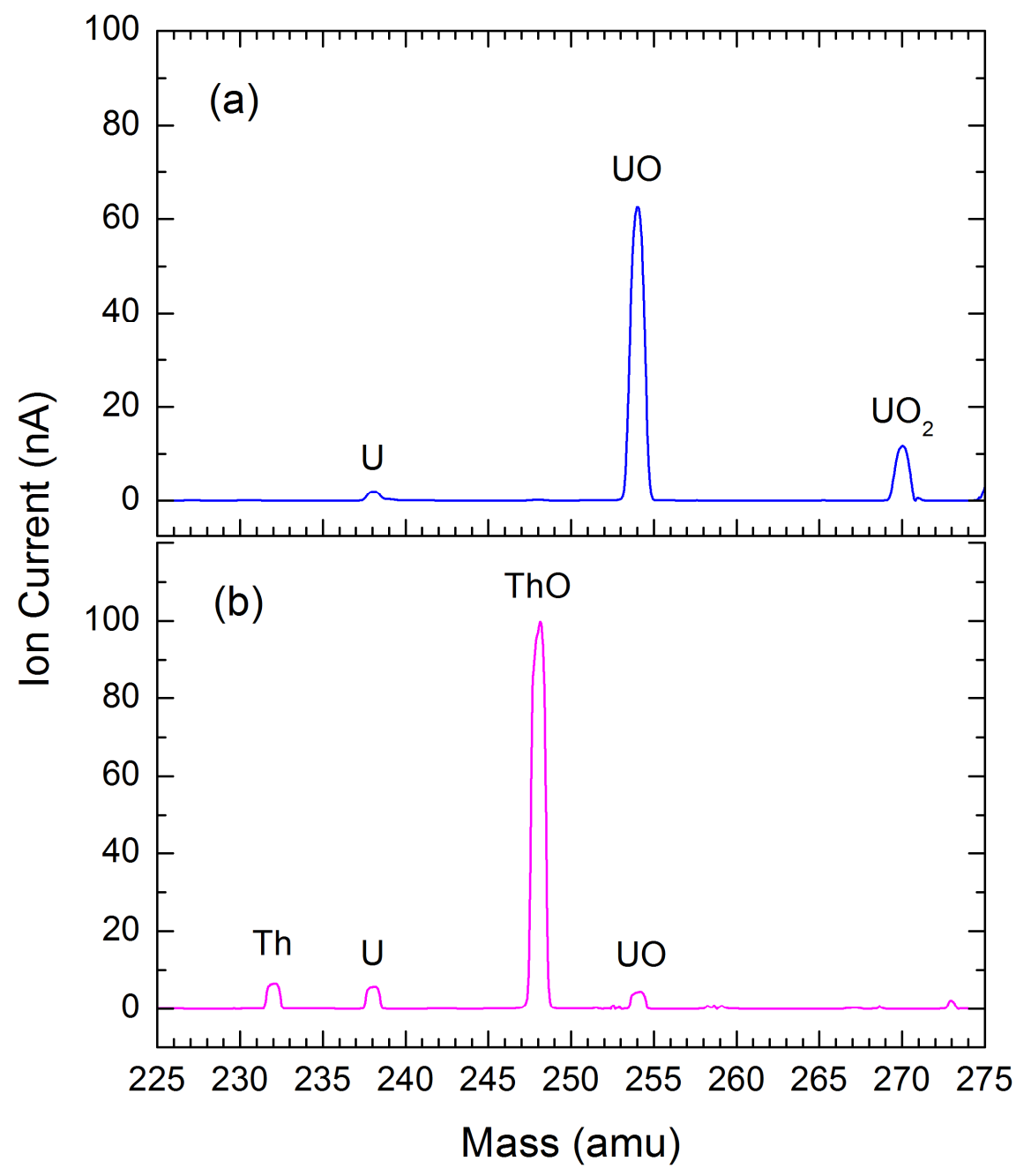

Figure 2 


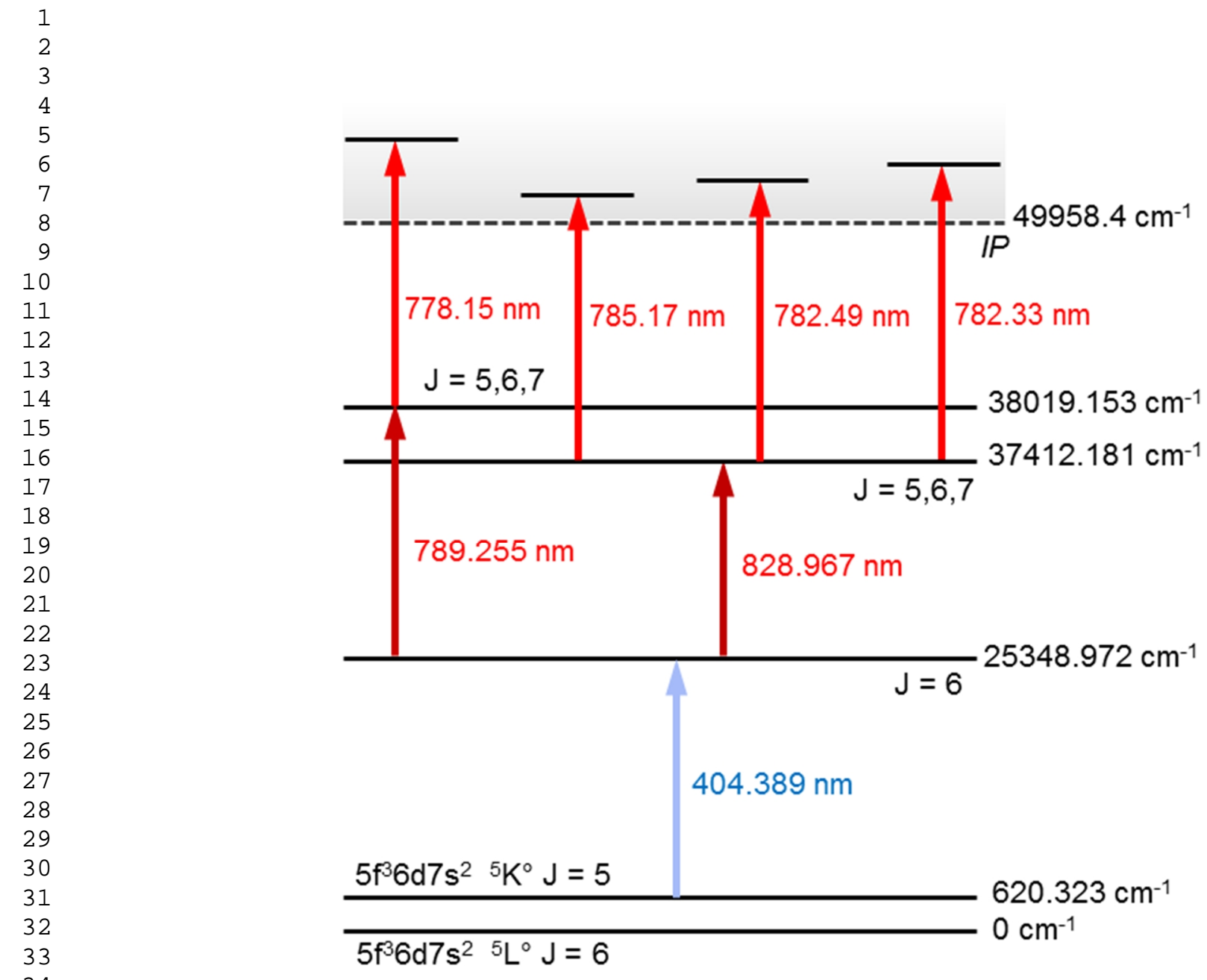

Figure 3 